\title{
Sagittal spino-pelvic balance is a crucial analysis for normal and degenerative spine
}

\author{
J. C. Le Huec $\cdot$ P. Roussouly
}

Received: 11 July 2011/Published online: 3 August 2011

(C) Springer-Verlag 2011

Human beings are the only vertebrates to maintain an upright, totally vertical, bipedal position submitted to the law of gravity. Unlike other vertebrates, the human spine comprises successive, opposing curves which allows the trunk to maintain an erect position. Lumbar lordosis is found in no other species. Bipedal locomotion in nonhuman primates is extremely limited. Those species often use their long forelimbs in pronation position to keep balance when trying to perform a bipedal displacement. Erect position is linked with pelvic rotation and enlargement, associated with modifications of the spine sagittal curvatures and muscle adaptation [1]. Human bipedalism is exclusive, stable and ergonomic. In order to maintain this posture for prolonged periods, the erect position should be economical in terms of energy expenditure. To correctly analyze the erect posture, it is necessary to define spinal and pelvic parameters, and to correlate those parameters with global parameters in full standing position.

Since Hippocrates provided a precise description of the segments and normal curves of the spine over 2000 years ago, clinicians have attempted to understand the mechanics underlying spinal balance [2]. For an individual to stand, it is necessary that the projection of the centre of the body mass on the ground plane lies behind the toes and is in front of the heels (the base of support). In order to maintain this

J. C. Le Huec $(\bowtie)$

Chief spine Unit 2, Bordeaux University Hospital Pellegrin,

Director Surg. Research Lab, Place Amélie Raba-Léon,

33076 Bordeaux, France

e-mail: j-c.lehuec@u-bordeaux2.fr

P. Roussouly

Department of Orthopedic Surgery, Centre de la Croix Rouge, CMCR des Massues, 92 rue Docteur Edmond Locard,

69000 Lyon, France position, for any individual, there will be an optimal combination of spinal, spino-pelvic and lower limb joint alignments, requiring the least muscle energy expenditure combined with the least discomfort, potentially caused by stretching of ligaments and joint capsules. Understanding the diversity of human spinal shape and orientation is pivotal to the understanding of the achievement of this optimal position, and to its restoration, in cases of sagittal imbalance. Despite ongoing interest in assessing the nature of the 'normal' curvature of the spine, there are no universally accepted parameters for its form $[5,6]$.

The hip joint, instead of being considered as a joint solely capable of allowing femoral movement against a fixed pelvis, should be considered as a joint that allows rotation of the pelvis against fixed femurs [5]. Rotation of the hip joint can, therefore, be considered as a fundamental factor that can interfere with the spinal shape through the pelvic anatomy.

The aims of this supplement are to review published data using the current techniques, to compare these different methods and to demonstrate that it is possible today to obtain clear parameters allowing a correct analysis of the sagittal plane of our substantial patient population. Case studies will be used to illustrate the importance of proper consideration of the thoracic kyphosis in relation to the lumbo-pelvic lordosis and the pelvic shape. Those cases will also demonstrate the importance of the degenerative process acting as a new parameter and its influence on modification of the spine balance.

The initial spino-pelvic organization will also interfere with the degenerative process of the spine [3]. The biomechanics of the spine differ according to the spino-pelvic shape [4]. Some spinal curvatures and spino-pelvic situations are more frequently subject to specific degenerative evolution [7]. As spine-aging process is also genetically 
driven, it is easy to understand that the degenerative process can vary dramatically from one patient to another leading to different imbalance situation with a similar spino-pelvic organization at the beginning.

Sagittal balance analysis, and the spino-pelvic organization linked to it, is a major tool when attempting to propose a diagnosis and a treatment for lumbalgia and degenerative spine disease. Spinal imbalance should be taken into consideration before initiation of any kind of treatment including conservative or surgical procedures.

Conflict of interest None.

\section{References}

1. Berge C (1998) Heterochronic processes in human evolution: an ontogenetic analysis of the hominid pelvis. Am J Phys Anthropol 105(4):441-459
2. Marketos SG, Skiadas P (1999) Hippocrates. The father of spine surgery. Spine 24(13):1381-1387

3. Gelb DE, Lenke LG, Bridwell KH, Blanke K, McEnery KW (1995) An analysis of sagittal spinal alignment in 100 asymptomatic middle and older aged volunteers. Spine 20(12):1351-1358

4. Berthonnaud E, Dimnet J, Roussouly P, Labelle H (2005) Analysis of the sagittal balance of the spine and pelvis using shape and orientation parameters. J Spinal Disord Tech 18(1):40-47

5. Boulay C, Tardieu C, Hecquet J, Benaim C, Mouilleseaux B, Marty C, Prat-Pradal D, Legaye J, Duval-Beaupère G, Pélissier J (2006) Sagittal alignment of spine and pelvis regulated by pelvic incidence: standard values and prediction of lordosis. Eur Spine J $15(4): 415-422$

6. Jackson RP, McManus AC (1994) Radiographic analysis of sagittal plane alignment and balance in standing volunteers and patients with low back pain matched for age, sex, and size. A prospective controlled clinical study. Spine 19(14):1611-1618

7. Barrey C, Jund J, Noseda O, Roussouly P (2007) Sagittal balance of the pelvis-spine complex and lumbar degenerative diseases. A comparative study about 85 cases. Eur Spine J 16:1459-1467 